\title{
Poor sleep quality and fatigue, but no excessive daytime sleepiness in myotonic dystrophy type 2
}

\author{
Alide A. Tieleman ${ }^{1}$ MD; Hans Knoop PhD; Anne-Els van de Logt ${ }^{1}$ MD; Gijs Bleijenberg ${ }^{2}$ PhD; \\ Baziel G.M. van Engelen ${ }^{1}$ MD, PhD; Sebastiaan Overeem ${ }^{1,3} M D, P h D$ \\ ${ }^{1}$ Department of Neurology, Donders Institute for Brain, Cognition and Behavior, Radboud University \\ Nijmegen Medical Centre, Nijmegen, the Netherlands \\ ${ }^{2}$ Expert Centre Chronic Fatigue, Radboud University Nijmegen Medical Centre, Nijmegen, the \\ Netherlands \\ ${ }^{3}$ Centre for Sleep Medicine 'Kempenhaeghe', Heeze, the Netherlands
}

\section{Corresponding author:}

S. Overeem, MD, PhD

Department of Neurology

Donders Institute for Brain, Cognition and Behavior

Radboud University Nijmegen Medical Centre

PO Box 9101, $6500 \mathrm{HB}$, Nijmegen, the Netherlands

Phone: $+31-24-3615202$

Fax: +31-24-3541122

E-mail: s.overeem@neuro.umcn.nl

Keywords: myotonic dystrophy type 2, sleep quality, fatigue, excessive daytime sleepiness, myotonic dystrophy type 1.

\section{Character count (title): 84}

Word count (excluding title page, abstract, references, figures and tables): 2742

The Corresponding Author has the right to grant on behalf of all authors and does grant on behalf of all authors, an exclusive licence (or non exclusive for government employees) on a worldwide basis to the BMJ Publishing Group Ltd and its Licensees to permit this article (if accepted) to be published in the Journal of Neurology, Neurosurgery \& Psychiatry editions and any other BMJPGL products to exploit all subsidiary rights, as set out in our licence (http://jnnp.bmijournals.com/ifora/licence.pdf)

. Conflict of interest: none declared. 


\section{ABSTRACT}

Background: In myotonic dystrophy type 1 (DM1), sleep disorders are common, with excessive daytime sleepiness (EDS) as a predominant feature. In myotonic dystrophy type 2 (DM2), the presence of sleep disturbances is unknown.

Objective: To investigate the frequency of EDS, poor sleep quality and fatigue in DM2.

Methods: We surveyed 29 genetically proven DM2 patients with the Epworth Sleepiness Scale (ESS), Pittsburgh Sleep Quality Index (PSQI) and Checklist Individual Strength (CIS). The results were compared with 29 adult-onset DM1 patients and 65 population controls, both matched for age and sex.

Results: Only $6.9 \%$ of DM2 patients had EDS compared to $44.8 \%$ of DM1 patients and $6.2 \%$ of population controls (DM2-DM1: $p=0.001$, DM2-controls: $p=0.51)$. Sleep quality was poor $(P S Q I>5)$ in both the DM2 and DM1 group, and differed significantly from population controls (DM2: $6.5 \pm 3.0$; DM1: $6.2 \pm 3.7$; controls $4.3 \pm 3.0$ ) (DM2-controls: $p=0.002$ ). Poor sleep quality was not explained by depression or other co-morbidity, but mainly due to sleep disturbances as a result of nocturnal pain. Comparable to the DM1 group, DM2 patients experienced severe fatigue (DM2: $38.7 \pm 13.1$; DM1: $42.9 \pm 8.5$; controls: $21.1 \pm 11.1$; DM2-controls: $p<0.001$ ). Results were not confounded by abnormal thyroid function or medication use.

Conclusion: These results provide new insight into the phenotype of DM2, and have consequences for clinical treatment. In addition, the absence of EDS in DM2 is a new discriminative feature with adult-onset DM1. 


\section{INTRODUCTION}

The myotonic dystrophies (DM) are dominantly inherited multisystem diseases, with muscle weakness and myotonia as the main neuromuscular symptoms. Other organs are prominently involved, with frequent early-onset cataracts, cardiac conduction abnormalities, gastro-intestinal symptoms, endocrine changes and central nervous system symptoms. ${ }^{1-3}$ Myotonic dystrophy type 1 (DM1) is the most common form of muscular dystrophy in adults, with a prevalence of $5-12$ per $100,000 .{ }^{1,4}$ The phenotype and genetics of myotonic dystrophy type 2 (DM2) have been described only recently, and its prevalence has not yet been established. ${ }^{5,6}$ The molecular basis of both DM1 and DM2 is a repeat instability in a noncoding part of a gene: a CTG-trinucleotide repeat in the DMPK gene on chromosome 19 (DM1) and a CCTG-tetranucleotide repeat in the ZNF9 gene on chromosome 3 (DM2). ${ }^{6,7}$ The resulting disruption of mRNA metabolism alters nuclear functions, including the splicing machinery. This leads to abnormal transcripts in many other genes, explaining the multisystemic nature of the diseases. $^{7}$

Interestingly, repeat instability in two unrelated genes causes comparable clinical phenotypes. In fact, DM2 was not recognized as a separate disorder until 1994. ${ }^{5,8}$ However, some recent studies suggest important differences in the clinical symptomatology of DM1 and DM2. For example, the severe congenital form encountered in DM1 is absent in DM2, and there is no confirmed evidence of anticipation in DM2.

Sleep disorders are very common in DM1, and are in fact considered a core feature. Of these, excessive daytime sleepiness (EDS) is a predominant symptom. ${ }^{1,9-11}$ EDS in DM1 can be severe, and has been described to resemble the sleepiness of primary hypersomnias such as narcolepsy. Besides daytime sleepiness, severe fatigue is present in up to $74 \%$ of adult-onset DM1 patients. ${ }^{12}$

In contrast to DM1, virtually nothing is known about sleep and fatigue in DM2. Some authors have suggested a comparable presence of daytime sleepiness in DM2, ${ }^{13,14}$ but systematic studies are lacking. We therefore performed a nationwide study into EDS, sleep quality, and fatigue in a cohort of DM2 patients, and compared these symptoms with both a matched group of adult-onset DM1 patients and with population controls. 


\section{METHODS}

\section{Subjects}

All known Dutch DM2 patients ( $\mathrm{N}=32$ ) were approached to participate. DM2 patients, and sex- and age-matched DM1 patients were retrieved from CRAMP, the Dutch neuromuscular database. ${ }^{15}$ Furthermore, 65 sex- and age-matched population controls were surveyed. All DM patients were above the age of 18 , were ambulatory, and had a genetically confirmed diagnosis. Exclusion criteria concerned the use of central nervous system stimulant medication or assisted ventilation such as nocturnal continuous positive airway pressure. The presence of a neuromuscular disorder was the only exclusion criterion for the population controls. All subjects gave written informed consent to participate, and the study was approved by the local ethical committee.

\section{Questionnaires}

Sleep quality

Nocturnal sleep quality was assessed using the Pittsburgh Sleep Quality Index (PSQI). ${ }^{16}$ The PSQI is a self-rated questionnaire that measures seven components of sleep over a 1-month time interval: subjective sleep quality, sleep latency, sleep duration, habitual sleep efficiency, sleep disturbances, use of sleeping medication and daytime dysfunction. Each component score has a range of 0-3 points. In all cases, a score of ' 0 ' indicates no difficulty, while a score of ' 3 ' indicates severe difficulty. The sum of the seven component scores is expressed as a global score, with a range of 0-21 points. The normal threshold is 5 ; higher scores indicate poorer sleep quality. ${ }^{16}$

\section{Excessive Daytime Sleepiness}

Daytime sleepiness was measured with the Epworth Sleepiness Scale (ESS). ${ }^{17}$ The ESS measures the tendency to fall asleep during eight different situations that are commonly encountered in daily life, yielding a score range of $0-24$. The upper limit of normal is 10 , and ESS scores above 10 are generally taken to reflect abnormal daytime sleepiness.

\section{Fatigue severity}

The presence of fatigue was assessed using the subscale 'fatigue severity' of the Checklist Individual 
Strength (CIS). ${ }^{18}$ The CIS measures the experience of fatigue-associated problems during the previous two weeks. The CIS-fatigue severity subscale contains eight items scored on a 7-point Likert scale. Scores can range between 8 and 56 ; higher scores indicate higher levels of fatigue and scores of 35 or more are considered to indicate severe fatigue. ${ }^{18}$

\section{Co-morbidity and medication use}

All participants filled out a standardized questionnaire regarding medication use. When available, thyroid function data was retrieved from the patients' medical files. Symptoms of depression were scored using the Beck Depression Inventory for primary care (BDI-pc). This shortened version of the BDI has seven items and includes cognitive and affective symptoms. ${ }^{19}$ Somatic symptoms of depression have been removed, to avoid overlap when used for patients with e.g. neuromuscular disorders. A score of 4 or more is indicative of clinical depression.

\section{Statistical analysis}

Numerical variables are shown as mean \pm standard deviation (range). Categorical data is shown as a number (percentage of total). One-way ANOVAs were used to compare the three study populations. Differences between DM2 and DM1 patients and between DM2 patients and population controls were then tested using t-test or chi-square test. Correlations were calculated with the Pearson coefficient. P-values below 0.05 were considered to be significant.

\section{RESULTS}

\section{Demography}

Of the 32 DM2 patients that were approached, 29 (91\%) responded and completed the questionnaires a home. DM2 patients originated from 12 unrelated families. A total of 34 matched DM1 patients were selected from the CRAMP database, 5 of which were excluded because of the use of modafinil $(n=4)$ or nocturnal continuous positive airway pressure $(n=1)$. The demographic characteristics of both patients groups and the population controls are listed in Table 1. 
The groups were well matched for age, sex and body mass index (BMI). Age-at-onset was identical for the DM groups. There were no differences between the three groups regarding the use of betablockers or sleeping medication. A quarter of DM2 patients used pain medication, as did $14 \%$ of DM1 patients and $5 \%$ of population controls (DM2 vs. controls $p=0.005$; DM2 vs. DM1 $p=0.32$ ). Thyroid function data was available for 28 DM2 patients and 14 DM1 patients; none of them suffered from thyroid dysfunction. Depression scores were higher in both DM groups compared with controls (BDI-pc score DM2: $2.2 \pm 2.7$; DM1: $2.3 \pm 3.1$; controls: $0.9 \pm 1.7$ ) (DM2 vs. DM1: $p=0.86$; DM2 vs. controls: $\mathrm{p}=0.006)$.

\section{Sleep quality}

Both DM2 and DM1 patients had significantly higher PSQI global scores than population controls, indicating a decrease in sleep quality (Table 2, Figure 1). Sixty-six percent of the DM2 group showed a poor sleep quality (PSQI >5) compared to $45 \%$ in the DM1 group and $26 \%$ in controls. (DM2 vs. controls: $p<0.001$, DM2 vs. DM1: $p=0.11$.

DM2 patients showed worst on the PSQI component subjective sleep quality, which differed significantly from population controls (See Table 3 for PSQI component scores). In addition, DM2 patients differed significantly from both DM1 patients and population controls regarding the PSQI component score sleep disturbances (Table 3). Pain was the most commonly reported cause for sleep disturbances in the DM2 group. Sixty-nine percent of DM2 patients reported pain as the cause of having had trouble sleeping during the past month, versus $34 \%$ of DM1 patients and $17 \%$ of controls (DM2 vs. DM1: $p=0.009$ and DM2 vs. controls: $p<0.001$ ). The PSQI component score daytime dysfunction was significantly higher in both DM2 and DM1 patients compared with population controls. The component scores on sleep duration, sleep efficiency and the use of sleeping medication did not differ significantly between the three groups (Table 3).

The sleep latency in the DM2 group (28.5 \pm 26.1 minutes, range 2-120) was comparable with the sleep latency in the DM1 group $(25.1 \pm 26.4$ minutes, range $2-120, p=0.63)$, both significantly longer than population controls $(15.2 \pm 12.6$, range $3-60, p=0.001)$. Fifty-two percent of DM2 patients 
reported to fall asleep within 30 minutes, compared to $59 \%$ in the DM1 group and $83 \%$ in the population control group. Total sleep duration was not different in the three groups.

PSQI scores were not correlated with age, age at onset or disease duration. Furthermore, there was no association between depression or fatigue scores and PSQI total score in DM2 patients (DM2: $r=0.29, p=0.13 ; r=0.35, p=0.06$ respectively). In DM1 patients and controls, small but significant associations were found between the BDI-pc and PSQI (DM1: $r=0.53, p=0.003$; controls: $r=0.29$, $\mathrm{p}=0.02)$.

\section{Excessive daytime sleepiness}

Patients with DM2 did not have elevated ESS scores compared to population controls, and the percentage of DM2 patients with EDS was also in the control range (Table 2). One DM2 patient had an ESS just above the cut-off (11), one DM2 patient reported severe EDS, with an ESS of 20. This latter patient also had poor nocturnal sleep quality (PSQI 11), experienced severe fatigue (CIS-fatigue 56) and symptoms of depression (BDI-pc 5). As expected, DM1 patients showed a significantly higher ESS score than population controls. Forty-five percent of DM1 patients showed significant EDS (ESS $>10$ ), compared to $6 \%$ of the population controls (Table 2, Figure 1). Significant associations were found between depression scores and EDS in DM2 and controls (DM2: $r=0.42, p=0.03$; DM1: $r=0.33$, $p=0.08$; controls: $r=0.42, p=0.001)$. ESS scores were not correlated with age, age at onset or disease duration.

\section{Fatigue severity}

DM2 patients experienced high levels of fatigue, with $66 \%$ reporting severe fatigue (Table 2, Figure 1 ). This was also the case for DM1 patients with over 85\% reporting severe fatigue (DM2 vs. DM1: $p=$ 0.066). Both DM groups had significantly higher CIS-fatigue scores than population controls (Table 2, Figure 1). As with daytime sleepiness, fatigue correlated with depression scores on the BDI-pc in DM2 and controls (DM2: $r=0.47, p=0.01$; DM1: $r=0.21, p=0.27$; controls: $r=0.52, p<0.001$ ). Other demographic variables were not associated with fatigue, except a worsening of fatigue with age in DM1 ( $r=0.57, p=0.001)$. 


\section{DISCUSSION}

We estimated the prevalence of poor sleep quality, daytime sleepiness and fatigue in patients with DM2, and compared these to adult-onset DM1 patients and population controls. DM2 patients showed significantly worse sleep quality than population controls. High levels of fatigue were equally present in both types of DM. In striking contrast to DM1 -in which EDS is a predominant feature- we found that daytime sleepiness is not part of the clinical picture of DM2.

DM2 and DM1 patients reported longer nocturnal sleep latencies than population controls. For DM1, this has been reported before. Laberge et al. found that only $47 \%$ of DM1 patients fell asleep within 30 minutes versus $76 \%$ of healthy controls, ${ }^{20}$ although recent polysomnographic (PSG) data by this research group revealed a mean sleep latency of about 15 minutes in 43 DM1 patients. ${ }^{21}$ The reason for the sleep onset insomnia in DM2 is unknown, and no PSG data of DM2 patients exists at the moment. It is likely that nocturnal myalgic pain is one of the influencing factors. Nocturnal sleep disturbances occurred significantly more often in DM2 than in DM1 or controls, and nocturnal pain was reported to be the most common cause. The prevalence of nocturnal awakenings because of pain was twice as high in DM2 compared with DM1. This high prevalence occurred despite the fact that almost a quarter of DM2 patients received pain medication at the time of study, and $10 \%$ used hypnotics. Previous studies reported musculoskeletal pain to be a frequent symptom of DM2, and in one-third of patients, pain was even the most disabling symptom. ${ }^{22}$ The major pain type associated with DM2 is exercise-induced, with relief at rest. However, besides episodic pain, pain does persist for more than 6 months in many patients, indicating chronic pain. ${ }^{22}$ This is compatible with our finding of significant and sleep-disturbing nocturnal pain.

The mean ESS scores in patients with DM2 were identical to the population controls and comparable to previously reported normal values. ${ }^{17}$ Furthermore, the prevalence of excessive daytime sleepiness $(E S S>10)$ in DM2 patients was equal to population controls. We confirmed the high prevalence of excessive daytime sleepiness in patients with DM1. ${ }^{21,23,24}$ We excluded DM1 patients who were using psychostimulants for sleepiness, perhaps selecting patients with a relatively mild sleepiness. Therefore, the difference in the prevalence of sleepiness in DM2 and DM1 may even be 
underestimated in our study. The reason for this difference remains unclear. It may be an indication for relatively minor involvement of the central nervous system in DM2, as has been suggested before. ${ }^{3}$ Further study into the differential molecular pathophysiology of both disorders may also yield new insights in the pathogenesis of hypersomnia. It is unclear why the DM1 patients with EDS were not taking psychostimulants. As there was no obvious contraindication to the use of psychostimulants in the DM1 patient group, this may have reflected differences in clinical practice by the treating physician or patient decision.

The low prevalence of EDS, despite high levels of fatigue, underscores the fact that these are clearly different symptoms. ${ }^{24}$ DM2 patients demonstrated high levels of fatigue, comparable with DM1. The proportion of DM2 patients with severe fatigue $(66 \%)$ is also similar to figures reported for other neuromuscular disorders such as facioscapulohumeral dystrophy (61\%) or hereditary motor and sensory neuropathy type 1 (64\%). ${ }^{12}$ Fatigue was not explained by medication use such as betablockers, or abnormal thyroid function.

Depression scores in both DM2 and DM1 were higher than in population controls. This has been repeatedly reported by others in DM1. ${ }^{23,25,26}$ However, most of these studies were before the advent of DNA-based diagnosis and probably included a number of DM2 patients. Meola et al. investigated both DM1 and DM2 patients and did not find a higher frequency of clinical depression in either. ${ }^{14}$ This difference may in part be explained by the smaller number of patients in their study and differences in study methodology (self-report versus structured interview). In the DM2 group as well as the controls, there were correlations between depression, sleepiness and fatigue scores. This is likely to reflect the fact that small increases in the ESS may not be specific for hypersomnia. The much higher ESS scores in DM1 patients point to more severe hypersomnia related to central nervous system dysfunction, which may occur independently of depression or fatigue. Depression was associated with sleepiness and fatigue scores in the DM2 group, and should be included in the differential diagnosis when DM2 patients experience hypersomnia.

We approached all known Dutch DM2 patients, from 12 families. The nationwide study combined with the high response rate of $91 \%$ makes the presence of a selection bias in the DM2 group unlikely. 
Other strengths of this study are the DNA-based diagnosis of DM2 and DM1, the use of validated questionnaires and the consideration of comorbid depression and thyroid dysfunction. However, we are not informed about the range of CCTG- and CTG expansion on chromosome 3 and 21. Laberge et al found that DM1 patients with fatigue or EDS and fatigue had significantly longer CTG repeats than those with neither these symptoms. ${ }^{10}$ In DM2 however, phenotypical influence of the size of the CCTG-expansion -which is in part due to the somatic instability of the repeat- has not yet established. $^{27}$

In conclusion, the absence of EDS in DM2 is another clinical feature that differentiates DM2 from DM1, in addition to absence of a severe congenital form and genetic anticipation. We have shown a decrease in the quality of sleep in patients with DM2. This poor sleep quality was not explained by depression or other co-morbidity, but mainly due to increased sleep latency and sleep disturbances. Nocturnal awakenings due to pain were the most common symptom mentioned. Fatigue is another commonly experienced symptom in DM2, and is not correlated with the use of beta-blockers, thyroid dysfunction or a poor sleep quality.

Future studies should assess the nature of the nocturnal sleep disruption in DM2 by polysomnographic assessment, for example to delineate the possible role of sleep related breathing disorders. Multiple sleep latency testing in DM2 patients would be less likely to demonstrate abnormalities in the absence of subjective daytime sleepiness on the ESS but may still be of value to perform as a more objective assessment. Future studies aiming to improve nocturnal sleep quality in DM2, may also assess the consequences on potential daytime sequalae such as depression, fatigue or health-related quality of life.

Our findings may have important clinical consequences. In view of the negative impact of sleep disruption on the quality of life of DM2 patients, we would support a low threshold for screening for sleep disturbances -especially related to nocturnal myalgia-, and for performing sleep studies. In view of the findings in the current study, pain management throughout the day and night may improve sleep and quality of life in DM2 patients. 


\section{ACKNOWLEDGMENTS}

We wish to thank the patients and controls for participating in this study and Dr. G. Borm for statistical assistance. 


\section{REFERENCES}

1. Harper,P.S., van Engelen,B.G., Eymard,B., and Wilcox,D.E. (2004): Myotonic Dystrophy: present management, future therapy. Oxford University Press, New York.

2. Tieleman AA, van VJ, Jansen JB, van der Kooi AJ, Borm GF, et al. Gastrointestinal involvement is frequent in Myotonic Dystrophy type 2. Neuromuscul Disord 2008;18:646-9.

3. Meola $G$ and Sansone V. Cerebral involvement in myotonic dystrophies. Muscle Nerve 2007;36:294-306.

4. Norwood FL, Harling C, Chinnery PF, Eagle M, Bushby K, et al. Prevalence of genetic muscle disease in Northern England: in-depth analysis of a muscle clinic population. Brain 2009;132:3175-86.

5. Ricker K, Koch MC, Lehmann-Horn F, Pongratz D, Otto M, et al. Proximal myotonic myopathy: a new dominant disorder with myotonia, muscle weakness, and cataracts. Neurology 1994;44:1448-52.

6. Liquori CL, Ricker K, Moseley ML, Jacobsen JF, Kress W, et al. Myotonic dystrophy type 2 caused by a CCTG expansion in intron 1 of ZNF9. Science 2001;293:864-7.

7. Brook JD, McCurrach ME, Harley HG, Buckler AJ, Church D, et al. Molecular basis of myotonic dystrophy: expansion of a trinucleotide (CTG) repeat at the $3^{\prime}$ end of a transcript encoding a protein kinase family member. Cell 1992;68:799-808.

8. Thornton CA, Griggs RC, and Moxley RT, III. Myotonic dystrophy with no trinucleotide repeat expansion. Ann Neurol 1994;35:269-72.

9. Hilton-Jones D. Myotonic dystrophy--forgotten aspects of an often neglected condition. Curr Opin Neurol 1997;10:399-401.

10. Laberge $L$, Dauvilliers $Y$, Begin $P$, Richer $L$, Jean $S$, et al. Fatigue and daytime sleepiness in patients with myotonic dystrophy type 1: to lump or split? Neuromuscul Disord 2009;19:397-402.

11. Park JD and Radtke RA. Hypersomnolence in myotonic dystrophy: demonstration of sleep onset REM sleep. J Neurol Neurosurg Psychiatry 1995;58:512-3.

12. Kalkman JS, Schillings ML, van der Werf SP, Padberg GW, Zwarts MJ, et al. Experienced fatigue in facioscapulohumeral dystrophy, myotonic dystrophy, and HMSN-I. J Neurol Neurosurg Psychiatry 2005;76:1406-9.

13. Moxley RT, III. 54th ENMC International Workshop: PROMM (proximal myotonic myopathies) and other proximal myotonic syndromes. 10-12th October 1997, Naarden, The Netherlands. Neuromuscul Disord 1998;8:508-18.

14. Meola G, Sansone V, Perani D, Scarone S, Cappa S, et al. Executive dysfunction and avoidant personality trait in myotonic dystrophy type 1 (DM-1) and in proximal myotonic myopathy (PROMM/DM-2). Neuromuscul Disord 2003;13:813-21. 
15. van Engelen BG, van VH, van Doorn PA, Faber CG, van der Hoeven JH, et al. The Dutch neuromuscular database CRAMP (Computer Registry of All Myopathies and Polyneuropathies): development and preliminary data. Neuromuscul Disord 2007;17:33-7.

16. Buysse DJ, Reynolds CF, III, Monk TH, Berman SR, and Kupfer DJ. The Pittsburgh Sleep Quality Index: a new instrument for psychiatric practice and research. Psychiatry Res 1989;28:193-213.

17. Johns MW. A new method for measuring daytime sleepiness: the Epworth sleepiness scale. Sleep 1991;14:540-5.

18. Prins JB, Bleijenberg G, Bazelmans E, Elving LD, de Boo TM, et al. Cognitive behaviour therapy for chronic fatigue syndrome: a multicentre randomised controlled trial. Lancet 2001;357:841-7.

19. Kalkman JS, Zwarts MJ, Schillings ML, van Engelen BG, and Bleijenberg G. Different types of fatigue in patients with facioscapulohumeral dystrophy, myotonic dystrophy and HMSN-I. Experienced fatigue and physiological fatigue. Neurol Sci 2008;29 Suppl 2:S238-S240

20. Laberge L, Begin P, Montplaisir J, and Mathieu J. Sleep complaints in patients with myotonic dystrophy. J Sleep Res 2004;13:95-100.

21. Laberge L, Begin P, Dauvilliers $\mathrm{Y}$, Beaudry $\mathrm{M}$, Laforte $\mathrm{M}$, et al. A polysomnographic study of daytime sleepiness in myotonic dystrophy type 1. J Neurol Neurosurg Psychiatry 2009;80:642-6.

22. George A, Schneider-Gold C, Zier S, Reiners K, and Sommer C. Musculoskeletal pain in patients with myotonic dystrophy type 2. Arch Neurol 2004;61:1938-42.

23. Phillips MF, Steer HM, Soldan JR, Wiles CM, and Harper PS. Daytime somnolence in myotonic dystrophy. J Neurol 1999;246:275-82.

24. van der WS, Kalkman J, Bleijenberg G, van EB, Schillings M, et al. The relation between daytime sleepiness, fatigue, and reduced motivation in patients with adult onset myotonic dystrophy. J Neurol Neurosurg Psychiatry 2003;74:138-9.

25. Bird TD, Follett $C$, and Griep E. Cognitive and personality function in myotonic muscular dystrophy. J Neurol Neurosurg Psychiatry 1983;46:971-80.

26. Duveneck MJ, Portwood MM, Wicks JJ, and Lieberman JS. Depression in myotonic muscular dystrophy. Arch Phys Med Rehabil 1986;67:875-7.

27. Day JW, Ricker K, Jacobsen JF, Rasmussen L, Dick KA, et al. Myotonic dystrophy type 2: molecular, diagnostic and clinical spectrum. Neurology 2003;60:657-64. 
Table 1. Demographic data of DM2 patients, DM1 patients and controls

\begin{tabular}{|c|c|c|c|c|c|}
\hline & DM2 & DM1 & Controls & $\begin{array}{l}\text { P-value } \\
\text { (DM2-DM1) }\end{array}$ & $\begin{array}{l}\text { P-value } \\
\text { (DM2-Controls) }\end{array}$ \\
\hline $\mathbf{N}$ & 29 & 29 & 65 & & \\
\hline Females & $20(71 \%)$ & $20(71 \%)$ & $42(65 \%)$ & & \\
\hline Age (yrs) & $53.2 \pm 12.1(28-71)$ & $52.8 \pm 12.1(28-72)$ & $52.4 \pm 13.7(26-73)$ & & \\
\hline BMI $\left(\mathrm{kg} / \mathrm{m}^{2}\right)$ & $25.1 \pm 3.8(18.8-36.0)$ & $25.6 \pm 3.3(16.5-30.9)$ & $25.5 \pm 4.0(15.4-39.7)$ & 0.613 & 0.662 \\
\hline Age of onset (yrs) & $35.9 \pm 13.4(12-67)$ & $37.3 \pm 13.2(15-61)$ & - & 0.695 & - \\
\hline Disease duration (yrs) & $17.3 \pm 15.0(2-57)$ & $15.7 \pm 9.0(1-38)$ & - & 0.612 & - \\
\hline Sleeping medication & $3(10 \%)$ & $5(17 \%)$ & $8(12 \%)$ & 0.446 & 0.638 \\
\hline Pain medication & $7(24 \%)$ & $4(14 \%)$ & $3(5 \%)$ & 0.315 & 0.005 \\
\hline Beta-blockers & $3(10 \%)$ & $2(7 \%)$ & $6(9 \%)$ & 0.640 & 0.865 \\
\hline
\end{tabular}

Data is shown as mean \pm SD (range) or $\mathrm{N}(\%)$. BMI = Body Mass Index. 
Table 2. PSQI, ESS and CIS-fatigue score in DM2, DM1 and controls

\begin{tabular}{|c|c|c|c|c|c|c|}
\hline & DM2 & DM1 & Controls & $\begin{array}{l}\text { P-value } \\
\text { (ANOVA) }\end{array}$ & $\begin{array}{l}\text { P-value } \\
\text { (DM2-DM1) }\end{array}$ & $\begin{array}{l}\text { P-value } \\
\text { (DM2-Controls) }\end{array}$ \\
\hline $\mathbf{N}$ & 29 & 29 & 65 & & & \\
\hline PSQI & $6.5 \pm 3.0$ & $6.2 \pm 3.7$ & $4.3 \pm 3.0$ & 0.003 & 0.785 & 0.002 \\
\hline$P S Q I>5$ & $19(65.5 \%)$ & $13(44.8 \%)$ & 17 (26.2\%) & 0.001 & 0.113 & $<0.001$ \\
\hline ESS & $4.3 \pm 4.5$ & $9.6 \pm 4.1$ & $5.4 \pm 3.9$ & $<0.001$ & $<0.001$ & 0.214 \\
\hline$E S S>10$ & $2(6.9 \%)$ & $13(44.8 \%)$ & $4(6.2 \%)$ & $<0.001$ & 0.001 & 0.508 \\
\hline CIS-fatigue & $38.7 \pm 13.1$ & $42.9 \pm 8.5$ & $21.1 \pm 11.1$ & $<0.001$ & 0.149 & $<0.001$ \\
\hline CIS-fatigue $\geq 35$ & $19(65.5 \%)$ & 25 (86.2\%) & $7(10.8 \%)$ & $<0.001$ & 0.066 & $<0.001$ \\
\hline
\end{tabular}

Data is shown as mean \pm SD or $\mathrm{N}(\%)$.

PSQI = Pittsburgh Sleep Quality Index, ESS $=$ Epworth Sleepiness Scale, CIS-fatigue $=$ Checklist Individual Strength-fatigue score. 
Table 3. Pittsburgh Sleep Quality Index (PSQI) component subscores in DM2, DM1 and controls

\begin{tabular}{lllllll}
\hline $\begin{array}{l}\text { PSQI component } \\
\text { DM2 }\end{array}$ & DM1 & Controls & $\begin{array}{l}\text { P-value } \\
\text { (ANOVA) }\end{array}$ & $\begin{array}{c}\text { P-value } \\
\text { (DM2-DM1) }\end{array}$ & $\begin{array}{c}\text { P-value } \\
\text { (DM2-Controls) }\end{array}$ \\
\hline Subjective sleep quality & $1.1 \pm 0.9$ & $0.9 \pm 0.7$ & $0.7 \pm 0.6$ & 0.024 & 0.270 & 0.008 \\
Sleep latency & $1.5 \pm 1.1$ & $1.0 \pm 1.1$ & $0.8 \pm 0.7$ & 0.010 & 0.147 & 0.001 \\
Sleep duration & $0.3 \pm 0.7$ & $0.5 \pm 0.7$ & $0.4 \pm 0.7$ & 0.831 & 0.578 & 0.597 \\
Sleep efficiency & $0.8 \pm 1.2$ & $1.0 \pm 1.2$ & $0.4 \pm 0.8$ & 0.016 & 0.449 & 0.075 \\
Sleep disturbances & $1.4 \pm 0.8$ & $1.0 \pm 0.7$ & $1.1 \pm 0.5$ & 0.017 & 0.039 & 0.008 \\
$\begin{array}{l}\text { Use of sleeping } \\
\text { medication }\end{array}$ & $0.1 \pm 0.4$ & $0.6 \pm 1.1$ & $0.3 \pm 0.8$ & 0.104 & 0.050 & 0.383 \\
Daytime dysfunction & $1.2 \pm 0.8$ & $1.2 \pm 0.9$ & $0.7 \pm 0.7$ & 0.001 & 0.877 & 0.002 \\
\hline
\end{tabular}

Data is shown as mean $\pm \mathrm{SD}$ or $\mathrm{N}(\%)$.

PSQI = Pittsburgh Sleep Quality Index 
Figure 1. Individual scores on the PSQI, ESS and CIS-fatigue. The cut-off for abnormal levels is indicated by a dotted line. 

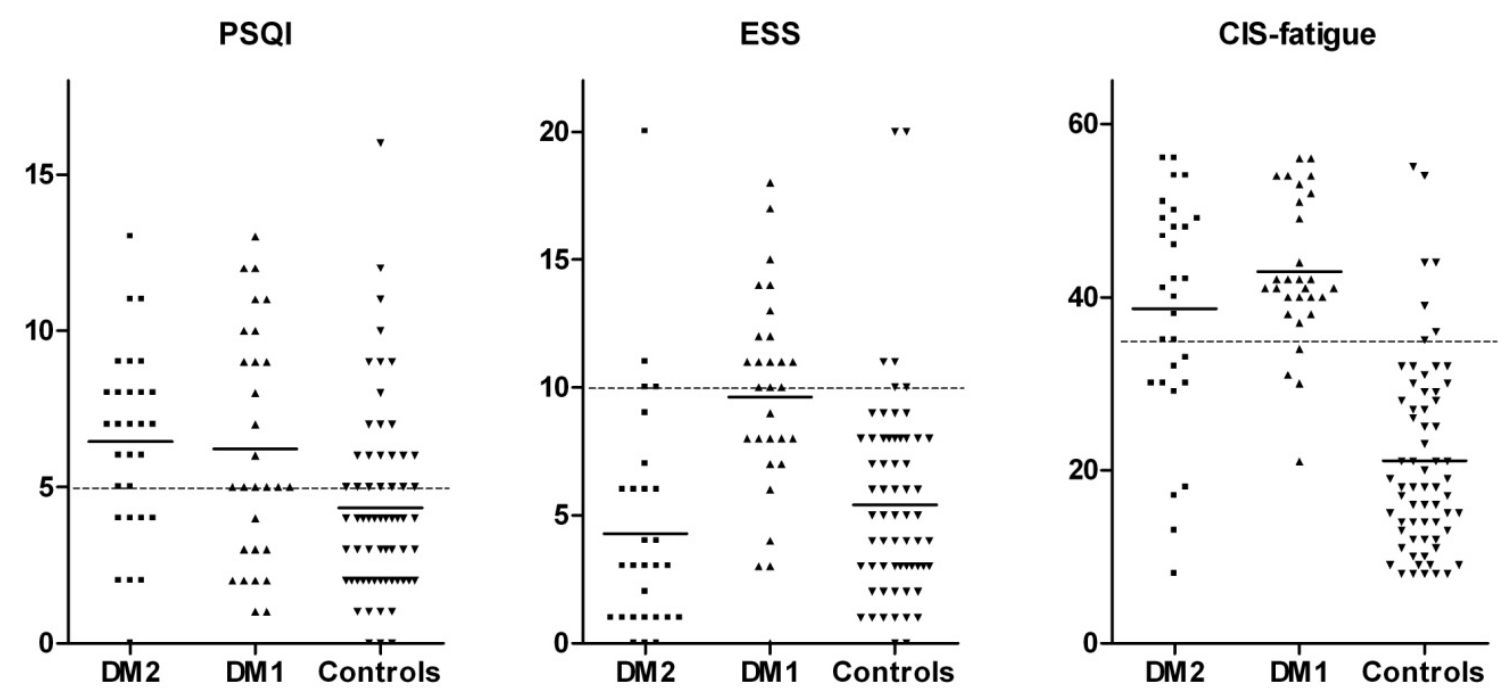